OPEN ACCESS

Edited by:

Yuling Qiu,

Tianjin Medical University, China

Reviewed by:

Hamed Barabadi,

Shahid Beheshti University of Medical

Sciences, Iran

Jung-Mao Hsu,

China Medical University, Taiwan

*Correspondence:

Yingjie Jia

jiayingjie1616@sina.com

these authors have contributed equally to this work

Specialty section:

This article was submitted to Pharmacology of Anti-Cancer Drugs,

a section of the journal

Frontiers in Pharmacology

Received: 12 July 2021 Accepted: 02 September 2021 Published: 13 September 2021

Citation:

Kong F, Wang C, Li X and Jia Y (2021) Kanglaite Combined With Epidermal

Growth Factor Receptor-Tyrosine Kinase Inhibitor Therapy for Stage III/IV

Non-Small Cell Lung Cancer: A

PRISMA-Compliant Meta-Analysis.

Front. Pharmacol. 12:739843.

doi: 10.3389/fphar.2021.739843

\section{Kanglaite Combined With Epidermal Growth Factor Receptor-Tyrosine Kinase Inhibitor Therapy for Stage III/ IV Non-Small Cell Lung Cancer: A PRISMA-Compliant Meta-Analysis}

\author{
Fanming Kong ${ }^{1,2 \dagger}$, Chaoran Wang ${ }^{1,2,3 \dagger}$, Xiaojiang $\mathrm{Li}^{1,2}$ and Yingjie Jia ${ }^{1,2 *}$ \\ ${ }^{1}$ Department of Oncology, First Teaching Hospital of Tianjin University of Traditional Chinese Medicine, Tianjin, China, ${ }^{2}$ National \\ Clinical Research Center for Chinese Medicine Acupuncture and Moxibustion, Tianjin, China, ${ }^{3}$ Graduate School, Tianjin University \\ of Traditional Chinese Medicine, Tianjin, China
}

Objective: Kanglaite(KLT), a type of Chinese medicine preparation, is considered as an adjuvant therapeutic option for malignant cancer treatment. This study aimed to systematically investigate the efficacy and safety of the combination of KLT and epidermal growth factor receptor-tyrosine kinase inhibitor (EGFR-TKI) for the treatment of stage III/IV non-small cell lung cancer.

Methods: Randomized controlled trials (RCTs) that compared KLT plus EGFR-TKI with EGFR-TKI alone for the treatment of stage III/IV non-small cell lung cancer were reviewed. Literature searches (up to July 10, 2021) were performed on PubMed, Web of Science, Cochrane Library, Embase, ClinicalTrials.gov, China National Knowledge Infrastructure (CNKI), Wanfang Database, and the Chinese Scientific Journal Database. Two researchers independently assessed the risk of bias with the tool of Cochrane Collaboration. RevMan 5.3.0 was used in the analysis of the included trial data.

Results: 12 RCTs recruiting 1,046 patients with stage III/IV NSCLC were included. Results showed that compared with EGFR-TKI alone, KLT plus EGFR-TKI significantly increased the disease control rate (DCR) (odds ratio $[\mathrm{OR}]=3.26$; 95\% confidence interval $[\mathrm{Cl}]: 2.22-4.77$; $p<0.00001)$, the objective response rate (ORR) (OR=2.59; 95\% Cl:1.87-3.58; $p<0.00001)$ and Karnofsky performance status (KPS) (OR = 2.76; 95\% Cl:1.73-4.39; $p<0.00001)$. Furthermore, patient immunity was enhanced with KLT plus EGFR-TKI. The combined treatment increased the percentage of CD4 $+T$ cells (weighted mean difference [WMD]= 5.36; 95\% Cl:3.60-7.13; $p<0.00001)$, the CD4+/CD8 + ratio $(\mathrm{WMD}=0.18 ; 95 \% \mathrm{Cl}$ : $0.08-0.27 ; p=0.004$ ), and percentage of NK cells (WMD=4.84; 95\% Cl: 3.66-6.02; $p<$ $0.00001)$.With regard to drug toxicity, the occurrence rate of nausea and vomiting was significantly reduced by KLT plus EGFR-TKI (OR=0.37; 95\% Cl: 0.16-0.86; $p=0.02)$.

Conclusion: KLT plus EGFR-TKI was effective in treating stage II//V non-small cell lung cancer. Thus, its application in these patients is worth promoting. Additional double-blind, well-designed and multicenter RCTs are required to confirm the efficacy and safety of this treatment.

Keywords: EGFR-TKI, kanglaite, NSCLC, meta-analysis, stage III/IV 


\section{INTRODUCTION}

Lung cancer remains the leading cause of cancer-related death worldwide (Sung et al., 2021). Despite the recent remarkable progress in screening, diagnosis, and treatment. 57\% of patients with lung cancer are diagnosed at an advanced or metastatic stage, during which the 5 -years relative survival rate is only $5 \%$ (Richards et al., 2017). Epidermal growth factor receptor-tyrosine kinase inhibitors (EGFR-TKIs) have improved clinical benefits for patients with metastatic non-small cell lung cancer (NSCLC) patients (Rosell et al., 2012). The National Comprehensive Cancer Network NSCLC Panel recommends identification of EGFR mutations for all patients with adenocarcinoma. EGFR-TKI combined with chemotherapy improved progression free survival (PFS) in untreated advanced NSCLC patients with EGFR mutation (Hosomi et al., 2020). However, the undesirable effects of EGFR-TKIs adversely affect the quality of life and treatment compliance of patients (Shah and Shah, 2019). Of patients with EGFR-mutated NSCLC, 20-40\% experience primary resistance to first or second-generation EGFR-TKIs, which is attributed to genetic alterations (Wang et al., 2016). Moreover, some patients do not show a good initial clinical response. Therefore, a EGFR-TKI-based combination treatment regimen may be more beneficial.

Traditional Chinese medicine (TCM) has been widely used as an adjuvant therapeutic option for cancer treatment (Xiang et al., 2019; Liu S.-H. et al., 2021). High-level clinical studies of TCM injection in cancer care has gradually increased (Yang et al., 2021). Kanglaite injection had been approved by the Chinese State Food and Drug Administration (SFDA) for the treatment of various malignant tumors. KLT is extracted and isolated from coix seeds (Coix lacryma-jobi). A few clinical studies on KLT for patients with solid tumor have been approved in the United States. KLT is the first TCM to be approved by the US Food and Drug Administration (FDA). The clinical mechanisms of KLT for NSCLC are related to the promotion of cancer cell apoptosis, inhibition of migration and proliferation and improvement of the immunity (Pan et al., 2012; Lu et al., 2013; Luo et al., 2017; Duan, 2018; Wu et al., 2018).

In previous studies, KLT injection combined with platinumbased chemotherapy showed significantly higher efficacy in the treatment of stage III/IV NSCLC (Hailang et al., 2020a; Li et al., 2020; Ni et al., 2021). With the widespread application of EGFRTKI, the number of published clinical studies on KLT combined with EGFR-TKI has been increasing. On the basis of previous clinical studies, we performed a PRISMA-compliant metaanalysis of KLT combined with EGFR-TKI in patients with stage III/IV NSCLC (Figure 1) to assess the clinical efficacy, quality of life, immune function (including percentages of $\mathrm{CD}^{+}$, $\mathrm{CD} 4^{+}$, NK cells and the CD4+/CD8+ratio) and adverse events. This work was conducted to provide comprehensive evidence for further studies and explore the clinical outcome of combination therapy with KLT and EGFR-TKI.

\section{MATERIALS AND METHODS}

We performed this meta-analysis following the Preferred Reporting Items for Systematic Reviews and Meta-Analyses (PRISMA) guidelines and Cochrane Handbook. The data were obtained from published trials. As this study does not involve animal and patient experiments, the ethical approval was not required.

\section{Literature Source and Search Strategy}

A comprehensive literature search was conducted by two independent researchers (FM Kong and CR Wang). Published studies were retrieved from eight databases including PubMed, Web of Science, Cochrane Library, Embase, ClinicalTrials.gov, the China National Knowledge Infrastructure (CNKI), Wanfang Database and the Chinese Scientific Journal Database. The last search date was July 10, 2021. In addition, we searched the relevant systematic reviews and meta-analyses to find the potential studies that may have been missed in the online searches.

The following search terms were used: "Lung Cancer" or "Non small cell lung cancer" or "NSCLC" or "Lung Carcinoma"or "Carcinoma of the lung" and "Kanglaite" and "Gefitinib" or "Erlotinib" or "Icotinib" or "Afatinib" or "Dacomitinib" or "Osimertinib" or "EGFR-TKI". No language limits were applied.

\section{Types of Studies and Selection Criteria}

All RCTs that compared KLT plus EGFR-TKI with EGFR-TKI alone were selected and assessed for inclusion in the study.

The inclusion criteria were as follows:

1) Randomized controlled trials (RCTs).

2) Patients with stage III/IV NSCLC confirmed by cytology or pathology.

3) Studies that included $>30$ patients with NSCLC.

4) Studies that compared the clinical outcomes of EGFR-TKI plus KLT adjuvant therapy (experimental group) with those of EGFR-TKI alone (control group).

5) The EGFR-TKIs included Gefitinib, Icotinib, Erlotinib, Afatinib, Dacomitinib, and Osimertinib.

The exclusion criteria were as follows:

1) Neither RCT nor "random"was mentioned.

2) Articles without sufficient data available.

3) Duplication of previous publications.

4) Case studies, review papers, comments, and conference abstracts.

\section{Data Extraction and Quality Assessment}

Data were independently extracted by two reviewers (FM Kong and CR Wang) according to the above inclusion and exclusion criteria; any disagreement was adjudicated by a third reviewer (XJ Li).

The following data were extracted:

1) The first author's name

2) Year of publication

3) Study location

4) Tumor stage

5) Number of cases

6) Age of the patients

7) Gender of the patients

8) Therapeutic regimens 


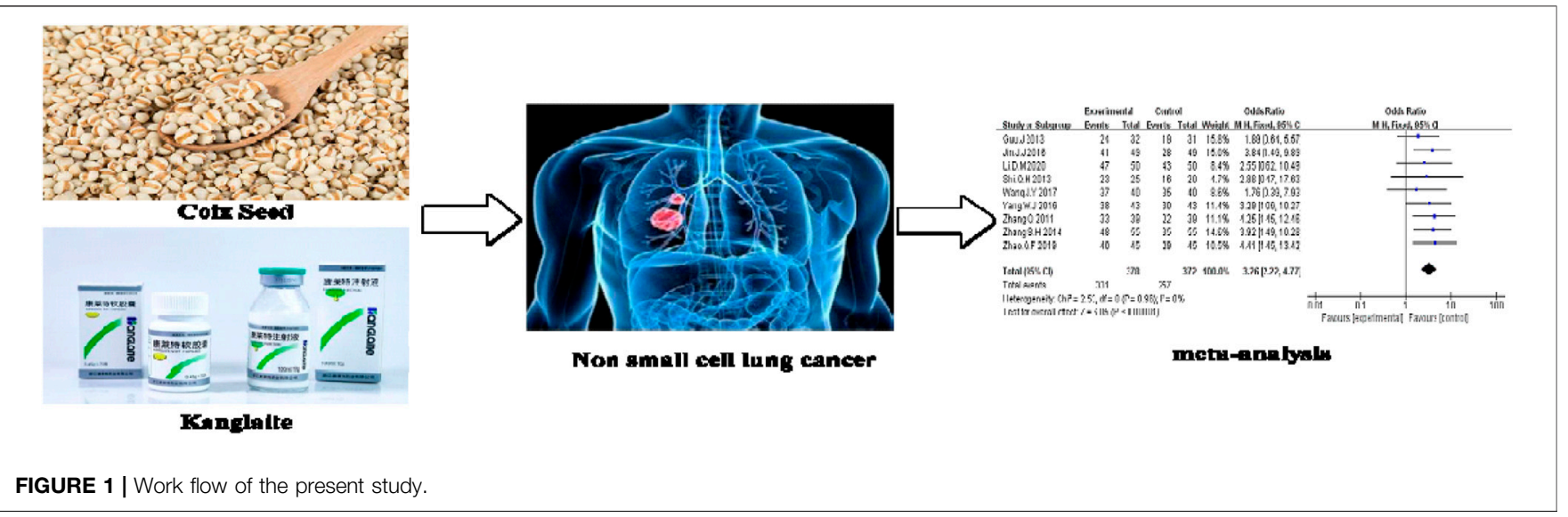

FIGURE 1 | Work flow of the present study.
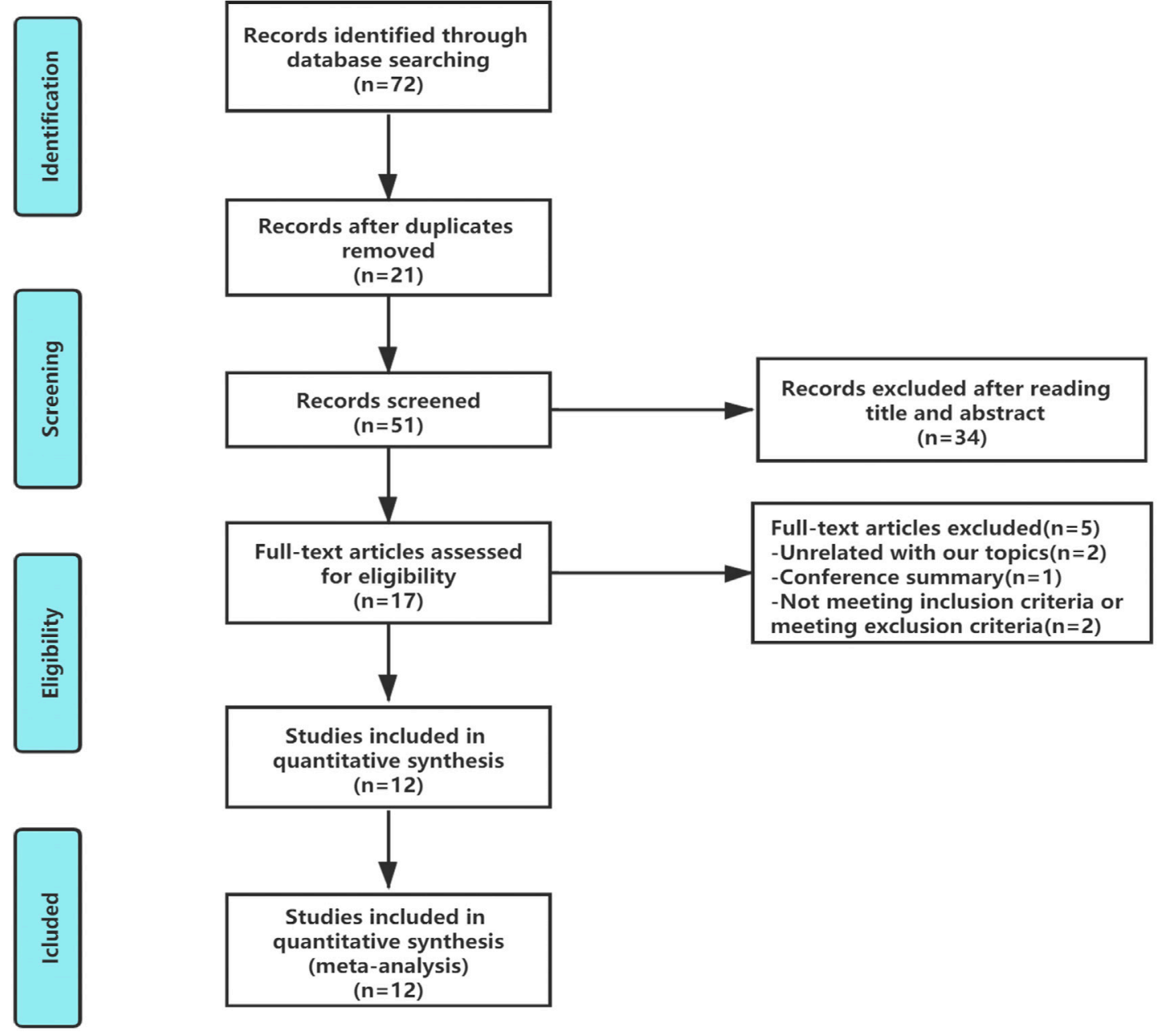

FIGURE 2 | Flow diagram showing study selection for the meta-analysis.

9) Main outcomes

\section{Outcome Definition}

The clinical responses assessed included treatment efficacy, performance status, immune function, and adverse events.
Treatment efficacy was evaluated in terms of the disease control rate (DCR), objective response rate (ORR), Karnofsky performance status (KPS), immune function indicators (percentages of $\mathrm{CD}^{+}$, $\mathrm{CD}^{+}, \mathrm{NK}$ cells and the CD4+/CD8+ratio) and adverse events including rash, nausea and vomiting, diarrhea, and liver injury. 
TABLE 1 | Clinical information from the eligible trials included in the meta-analysis.

\begin{tabular}{|c|c|c|c|c|c|c|c|c|}
\hline \multirow[t]{2}{*}{ Included studies } & \multirow[t]{2}{*}{ TNM stage } & \multirow{2}{*}{$\begin{array}{l}\text { Patients } \\
\text { Exp/Con }\end{array}$} & \multicolumn{2}{|c|}{ Therapeutic regimen } & \multirow{2}{*}{$\begin{array}{c}\text { Dosage } \\
\text { of kanglaite } \\
\text { (daily) }\end{array}$} & \multirow{2}{*}{$\begin{array}{c}\text { Dose of EGFR-TKI } \\
\text { (daily) (mg) }\end{array}$} & \multirow[t]{2}{*}{ Duration } & \multirow{2}{*}{$\begin{array}{l}\text { Outcomes } \\
\text { measure }\end{array}$} \\
\hline & & & Experimental & Control & & & & \\
\hline Ning.J.L (2015) & IIIB-IV & $93 / 93$ & $\mathrm{Con}+\mathrm{KLT}^{\mathrm{a}}$ & Gefitinib & $200 \mathrm{ml}$ & 250 & 8 weeks & IF \\
\hline Zhang.S.H (2014) & III-IV & $55 / 55$ & $\mathrm{Con}+\mathrm{KLT}^{\mathrm{a}}$ & Gefitinib & $200 \mathrm{ml}$ & 250 & 9 weeks & DCR ORR KPS AE \\
\hline Li.D.M (2020) & IIIB-IV & $50 / 50$ & $\mathrm{Con}+\mathrm{KLT}^{\mathrm{b}}$ & Gefitinib & $2.7 \mathrm{~g}$ Qid & 250 & $\begin{array}{l}\text { Not } \\
\text { provide }\end{array}$ & DCR ORR AE \\
\hline Wang.J.Y (2017) & IIIB-IV & $40 / 40$ & $\mathrm{Con}+\mathrm{KLT} \mathrm{T}^{\mathrm{b}}$ & Gefitinib & $2.7 \mathrm{~g}$ Qid & 250 & $60 \mathrm{~d}$ & DCR ORR AE \\
\hline Zeng.H.X (2014) & IIIB-IV & $23 / 23$ & $\mathrm{Con}+\mathrm{KLT}^{\mathrm{a}}$ & Gefitinib & $200 \mathrm{ml}$ & 250 & 6 weeks & IF AE KPS \\
\hline Yang.L (2016) & III-IV & $32 / 32$ & $\mathrm{Con}+\mathrm{KLT}^{\mathrm{a}}$ & Gefitinib & $200 \mathrm{ml}$ & 250 & 6 weeks & $\mathrm{IF}$ \\
\hline Yang.W.J (2016) & IIIB-IV & $43 / 43$ & $\mathrm{Con}+\mathrm{KLT}^{\mathrm{a}}$ & Icotinib & $200 \mathrm{ml}$ & 375 & 9 weeks & $\begin{array}{l}\text { DCR ORR KPS } \\
\text { IF AE }\end{array}$ \\
\hline Guo.J (2013) & IIIB-IV & $32 / 31$ & $\mathrm{Con}+\mathrm{KLT}^{\mathrm{a}}$ & Erlotinib & $200 \mathrm{ml}$ & 150 & 9 weeks & DCR ORR IF AE \\
\hline Jin.J.J (2016) & III-IV & $49 / 49$ & $\mathrm{Con}+\mathrm{KLT}^{\mathrm{a}}$ & Gefitinib & $200 \mathrm{ml}$ & 250 & 3 weeks & DCR ORR IF AE \\
\hline Zhang.Q (2011) & III-IV & $39 / 39$ & $\mathrm{Con}+\mathrm{KLT}^{\mathrm{a}}$ & Gefitinib & $100 \mathrm{ml}$ & 250 & 9 weeks & DCR ORR KPS AE \\
\hline Zhao.Q.F (2019) & III-IV & $45 / 45$ & $\mathrm{Con}+\mathrm{KLT}^{\mathrm{a}}$ & Gefitinib & $200 \mathrm{ml}$ & 251 & 3 weeks & DCR ORR IF AE \\
\hline Shi.Q.H (2013) & IIIB-IV & $25 / 20$ & $\mathrm{Con}+\mathrm{KLT}^{\mathrm{a}}$ & Gefitinib & $100 \mathrm{ml}$ & 250 & $60 \mathrm{~d}$ & DCR ORR KPS \\
\hline
\end{tabular}

Con, control group; Exp, experimental group; DCR, disease control rate; ORR, objective response rate; KPS, Karnofsky performance status; IF, Immune function; AE, adverse event. ${ }^{a}$ Kanglaite injection.

${ }^{b}$ Kanglaite capsule.

\begin{tabular}{|c|c|c|c|c|c|c|c|c|}
\hline Parameter & Factors at study & $\begin{array}{c}\text { Experimental } \\
\text { Patients(n) }\end{array}$ & $\begin{array}{c}\text { Control } \\
\text { Patients(n) }\end{array}$ & $\begin{array}{c}\text { Analysis } \\
\text { method }\end{array}$ & $\mathbf{I}^{2}$ & $\begin{array}{c}\text { Odds } \\
\text { ratio (OR) }\end{array}$ & $95 \% \mathrm{Cl}$ & $p$-value \\
\hline \multirow[t]{9}{*}{ DCR } & Sample size & & & & & & & \\
\hline & $\geq 80$ & 282 & 282 & Fixed & 0 & 3.44 & $2.17-5.44$ & $<0.00001$ \\
\hline & $<80$ & 96 & 90 & Fixed & 0 & 2.87 & $1.43-5.74$ & 0.003 \\
\hline & Form of KLT & & & & & & & \\
\hline & Injection & 288 & 282 & Fixed & 0 & 3.48 & $2.31-5.46$ & $<0.00001$ \\
\hline & Capsule & 90 & 90 & Fixed & 0 & 2.15 & $0.77-6.01$ & 0.14 \\
\hline & Duration & & & & & & & \\
\hline & $3 W$ & 94 & 94 & Fixed & 0 & 1.42 & $1.19-1.70$ & 0.0001 \\
\hline & $9 \mathrm{~W}$ & 169 & 168 & Fixed & 0 & 1.34 & $1.18-1.53$ & $<0.00001$ \\
\hline \multirow[t]{9}{*}{ ORR } & Sample size & & & & & & & \\
\hline & $\geq 80$ & 282 & 282 & Fixed & 0 & 2.96 & $2.01-4.37$ & $<0.00001$ \\
\hline & $<80$ & 96 & 90 & Fixed & 0 & 1.89 & $1.04-3.42$ & 0.04 \\
\hline & Form of KLT & & & & & & & \\
\hline & Injection & 288 & 282 & Fixed & 0 & 2.54 & $1.80-3.58$ & $<0.00001$ \\
\hline & Capsule & 90 & 90 & Fixed & 0 & 3.03 & $1.13-8.14$ & 0.003 \\
\hline & Duration & & & & & & & \\
\hline & $3 W$ & 94 & 94 & Fixed & 0 & 3.32 & $1.79-6.15$ & 0.0001 \\
\hline & $9 \mathrm{~W}$ & 169 & 168 & Fixed & 0 & 2.29 & $1.47-3.57$ & 0.0002 \\
\hline
\end{tabular}

\section{Risk of Bias Assessment}

Two researchers (XJ Li and YJ Jia) independently assessed the risk of bias with the tool of Cochrane Collaboration (Higgins et al., 2011). The Cochrane Collaboration contained the following assessment tools: random sequence generation; allocation concealment; blinding of participants, and personnel; blinding of outcome assessment; incomplete data; selective reporting and other bias. Each study was classified as "low risk of bias", "unclear risk of bias"or"high risk of bias". Any disagreement was settled through the third researcher (FM Kong).

\section{Statistical Analysis}

Statistical analysis was performed with the RevMan 5.3 software (Nordic Cochran Centre, Copenhagen, Denmark) software. Treatment effects were mainly represented by odds ratio (OR), and continuous data were shown as the weighted mean difference (WMD) with a $95 \%$ confidence intervals (CI). $p$ value $<0.05$ was considered statistically significant. Heterogeneity among the studies was assessed by Cochran's Q test; $\mathrm{I}^{2}<50 \%$ or $p>0.1$ indicated a lack of heterogeneity among the studies. When the level of heterogeneity was small $\left(\mathrm{I}^{2}<50 \%\right)$, a fixed-effects model was applied for estimation; otherwise, a random-effects model was selected.

\section{RESULTS}

\section{Search Results}

In total, 72 articles were initially identified. Of these articles, 21 papers were excluded because they were duplicates. After the title 
A

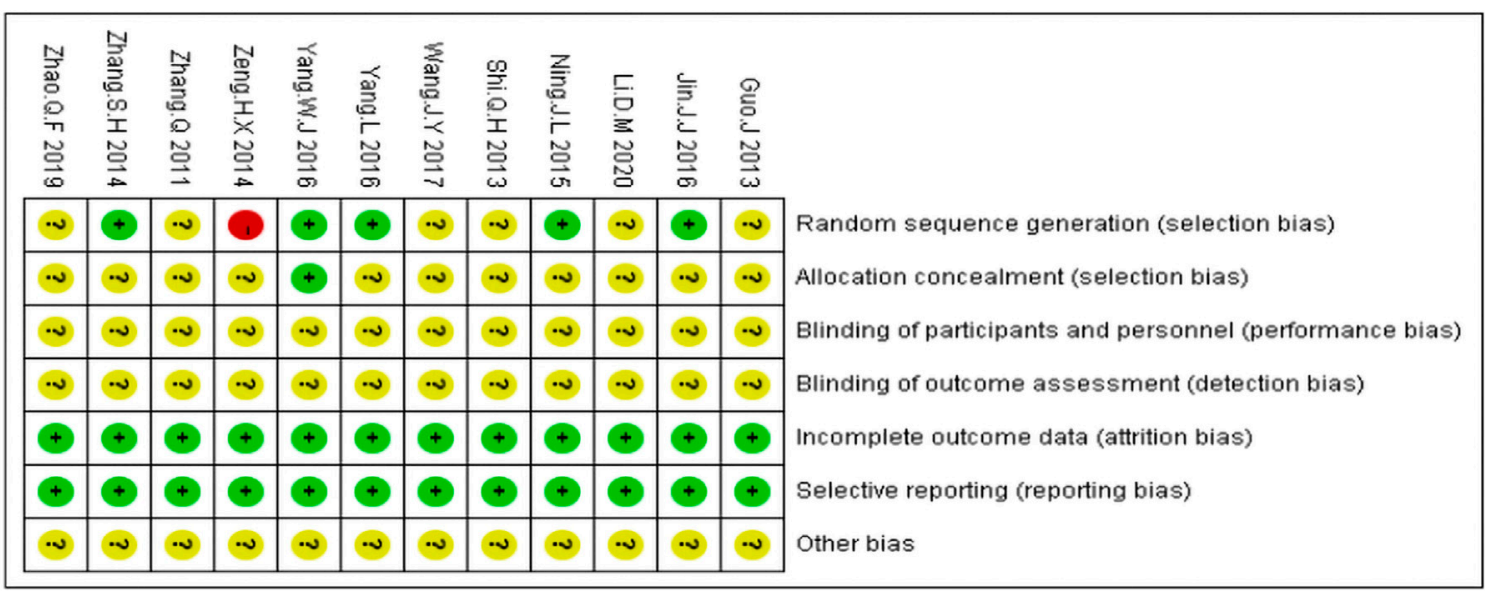

B

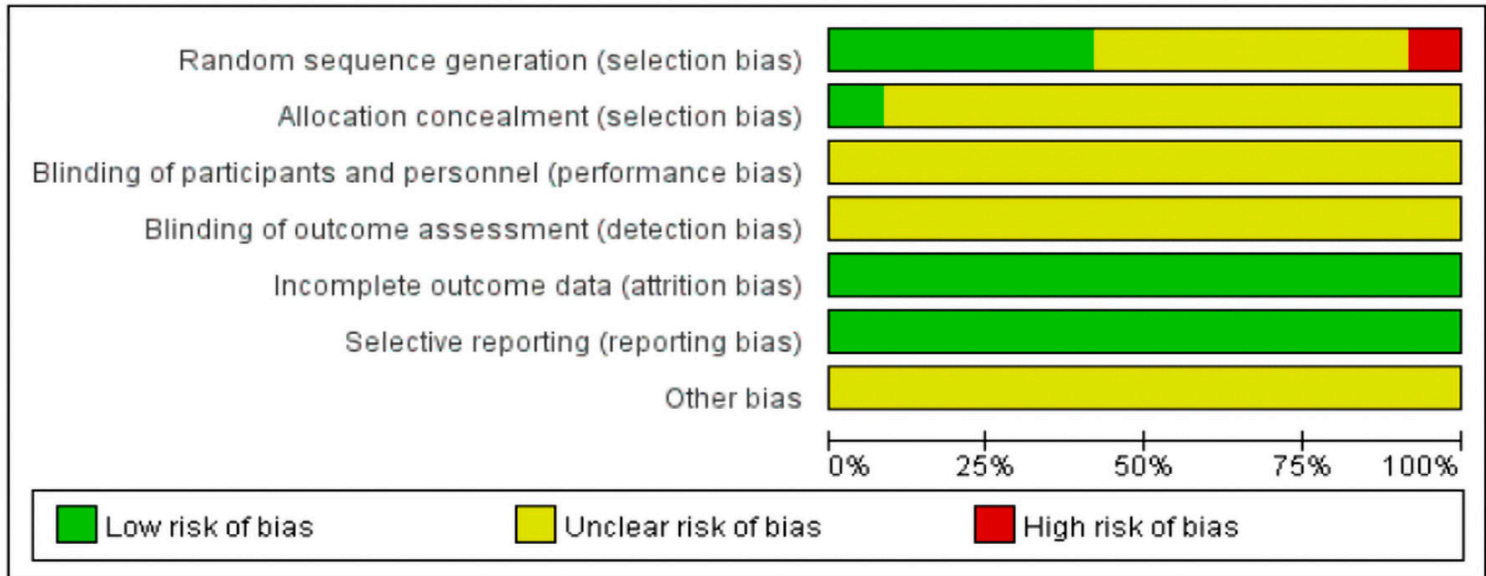

FIGURE 3 | Risk of bias of the included studies (A) Risk of bias graph (B) Risk of bias summary.

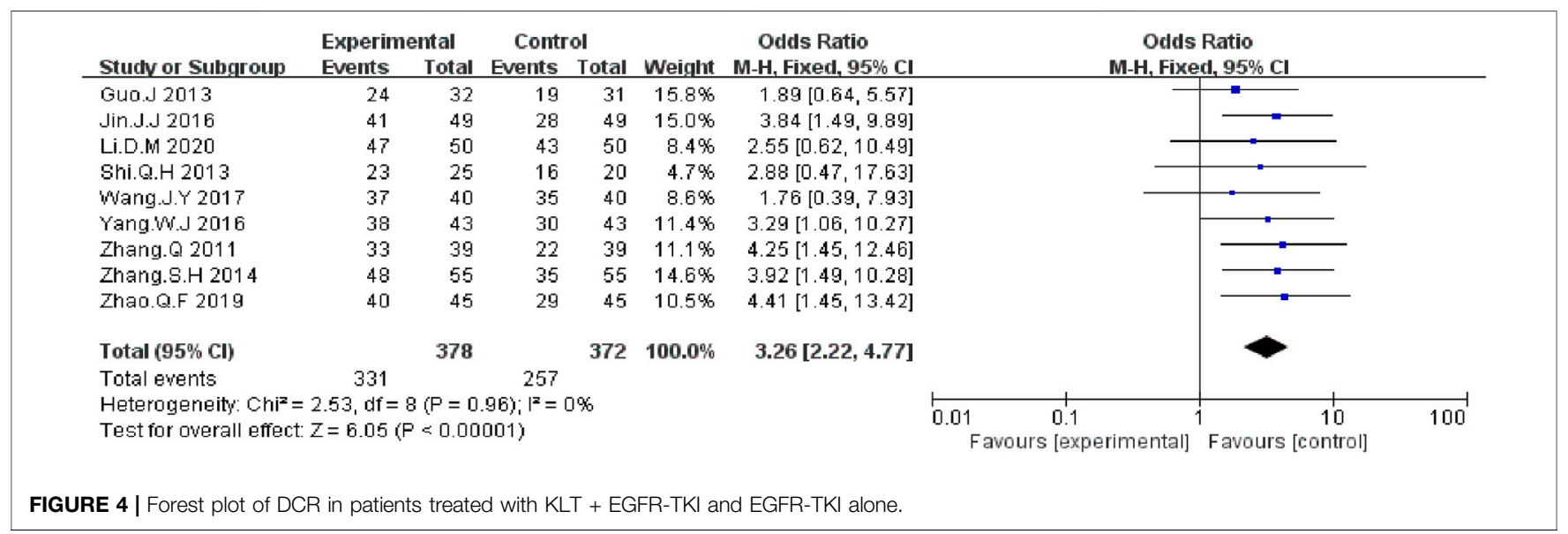

and abstract review, 34 articles were further excluded. After a detailed assessment of the full text articles, those unrelated with our topics $(n=2)$, conference summary $(n=1)$, and those that did not meeting the inclusion criteria or exclusion criteria $(n=2)$ were also excluded. Ultimately, 12 trials involving a total of 1,046 patients were included in this analysis (Figure 2). 


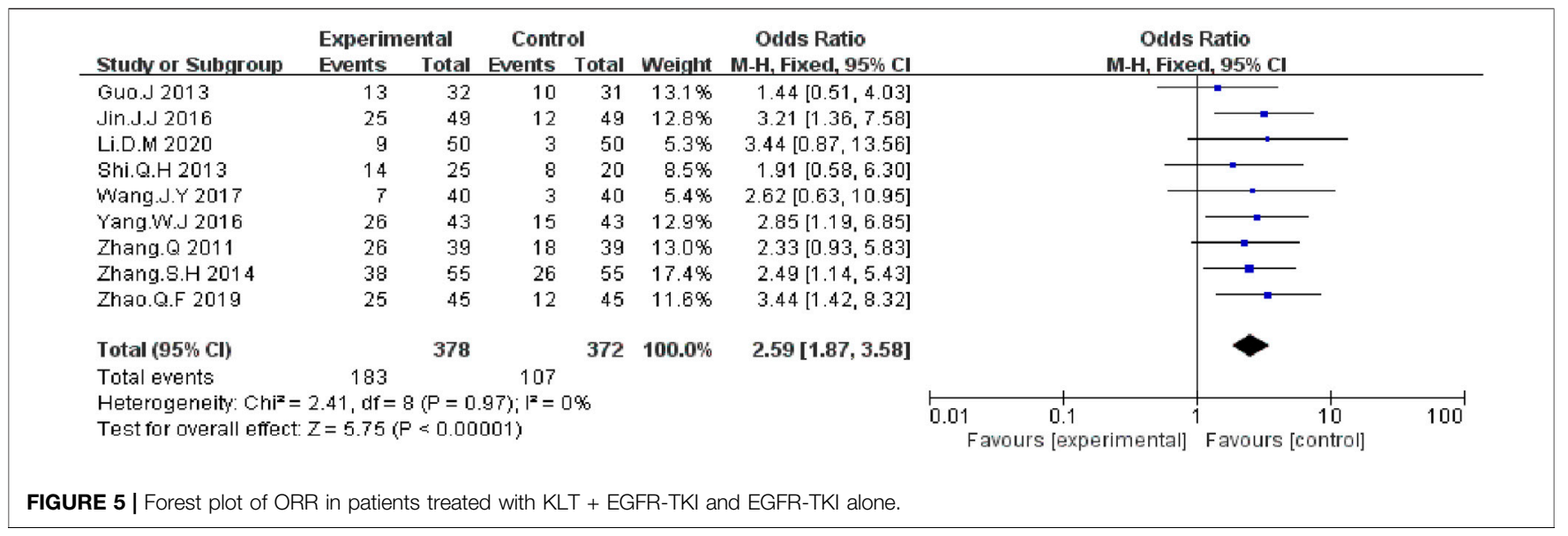

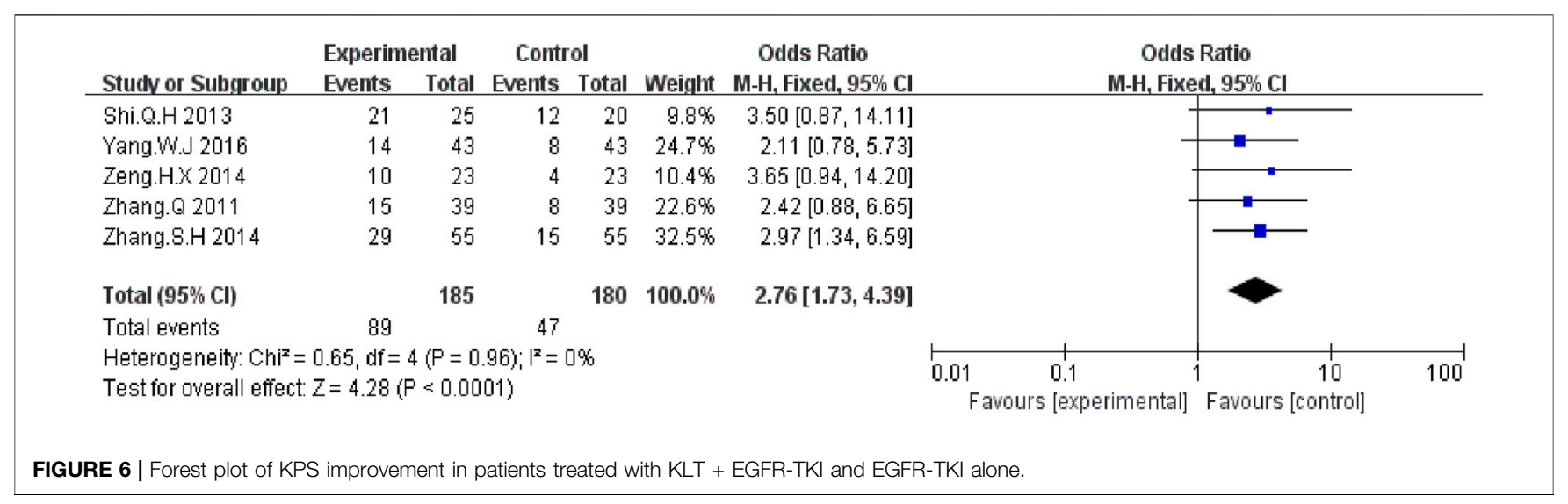

\section{Characteristics of Included Studies}

Table 1 summarizes the basic characteristics of the 12 RCTs. All the studies were conducted in different medical centers in China (Zhang and Yuan, 2011; Guo and Wang, 2013; Shi and Chen, 2013; Zeng et al., 2014; Zhang and Zhou, 2014; Ning et al., 2015; Jin et al., 2016; Yang, 2016; Yang et al., 2016; Wang et al., 2017; Zhao et al., 2019; Li, 2020). A total of 1,046 patients were recruited, of which 526 patients with stage III/IV NSCLC were treated with EGFR-TKI in combination with KLT, while 520 patients were treated with EGFR-TKI alone, with a sample size of 46-186 in each trial. The course of treatment lasted from 3 to 9 weeks. The control group received EGFR-TKIs, including gefitinib (Zhang and Yuan, 2011; Shi and Chen, 2013; Zeng et al., 2014; Zhang and Zhou, 2014; Ning et al., 2015; Jin et al., 2016; Yang et al., 2016; Wang et al., 2017; Zhao et al., 2019; Li, 2020), Icotinib (Yang, 2016) and Erlotinib (Guo and Wang, 2013). The patients in the experimental group received EGFRTKI in combination with KLT, including KLT injections (Zhang and Yuan, 2011; Guo and Wang, 2013; Shi and Chen, 2013; Zeng et al., 2014; Zhang and Zhou, 2014; Ning et al., 2015; Jin et al., 2016; Yang, 2016; Yang et al., 2016; Zhao et al., 2019) and KLT capsules (Wang et al., 2017; Li, 2020). Patient characteristics are shown in Table 1.

\section{Risk of Bias Assessment}

The bias risk analysis performed using the Cochrane Collaboration tool revealed bias in all the included studies. "Random" or "randomized" or "randomization" was mentioned in 11 studies, except for one study (Zeng et al., 2014). Only one study reported allocation concealment (Yang, 2016). All the included studies reported detailed outcome data. None of the 12studies provided blinding of the outcome data, clear descriptions of selective and other biases. Risk of bias assessment are shown in Figure 3.

\section{Outcomes Measures}

Disease Control Rate and Objective Response Rate

We extracted the data on DCR and ORR data from nine included RCTs report (Zhang and Yuan, 2011; Guo and Wang, 2013; Shi and Chen, 2013; Zhang and Zhou, 2014; Jin et al., 2016; Yang, 2016; Wang et al., 2017; Zhao et al., 2019; Li, 2020). The meta-analysis data showed that, compared with EGFR-TKI alone, KLT plus EGFR-TKI significantly improved DCR (OR $=3.26 ; 95 \%$ CI:2.22-4.77; $p<0.00001)$ (Figure 4) and ORR (OR: 2.59; 95\% CI: 1.87-3.58; $p<0.00001$ ) (Figure 5). Statistical homogeneity was observed for both outcomes $\left(\mathrm{I}^{2}=0 \%\right)$. 


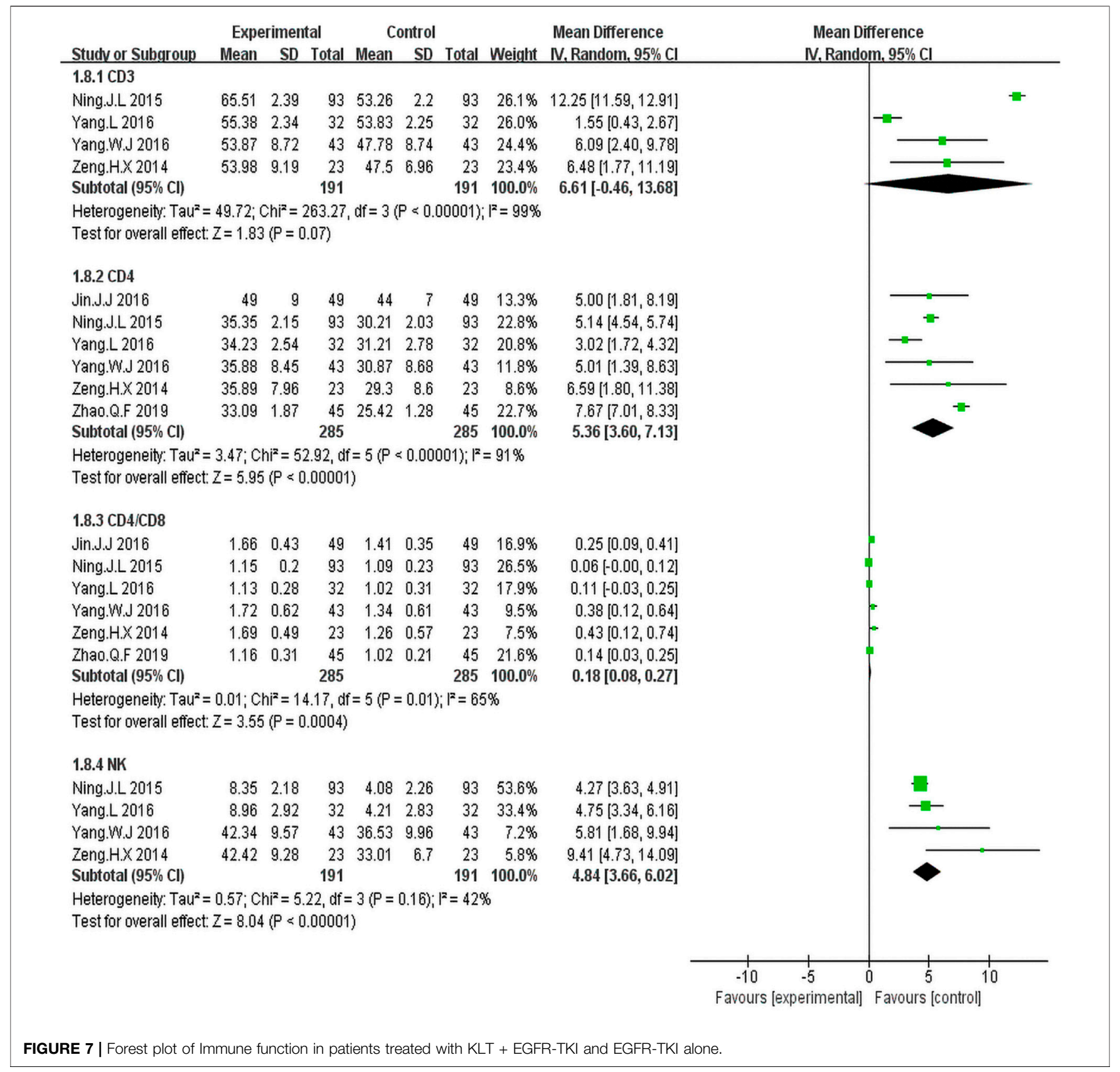

\section{Karnofsky Performance Status}

Five trials (Zhang and Yuan, 2011; Shi and Chen, 2013; Zeng et al., 2014; Zhang and Zhou, 2014; Yang, 2016) with a total of 750 patients reported improvement in KPS improvement. According to the results, the KPS was significantly higher in the KLT plus EGFR-TKI group than the control group (OR $=2.76,95 \% \mathrm{CI}$ : 1.73-4.39; $p<0.00001$ ) (Figure 6). A fixed-effect model was used due to the heterogeneity $\left(\mathrm{I}^{2}=0 \%\right)$.

\section{Immune Function}

Four trials (Zeng et al., 2014; Ning et al., 2015; Yang, 2016; Yang et al., 2016) including 382 patients reported percentages of $\mathrm{CD}^{+}$cells. Statistical heterogeneity was observed $\left(\mathrm{I}^{2}=99 \%\right.$, $p<0.00001$ ); Hence, the random effect model was employed. The results showed that the percentage of $\mathrm{CD}^{+}$cells was similar between the KLT plus EGFR-TKI group and control group $(\mathrm{WMD}=6.61 ; 95 \%$ CI: 0.46 to $13.68 ; p=0.07$ ) (Figure 7).

Six trials (Zeng et al., 2014; Ning et al., 2015; Jin et al., 2016; Yang, 2016; Yang et al., 2016; Zhao et al., 2019) including 570 patients reported percentage of $\mathrm{CD} 4+$ cells and the $\mathrm{CD} 4+$ / $\mathrm{CD} 8+$ ratio.There was statistical heterogeneity. The random effect model was employed for the analysis. The results illustrated that KLT plus EGFR-TKI group had an advantage 


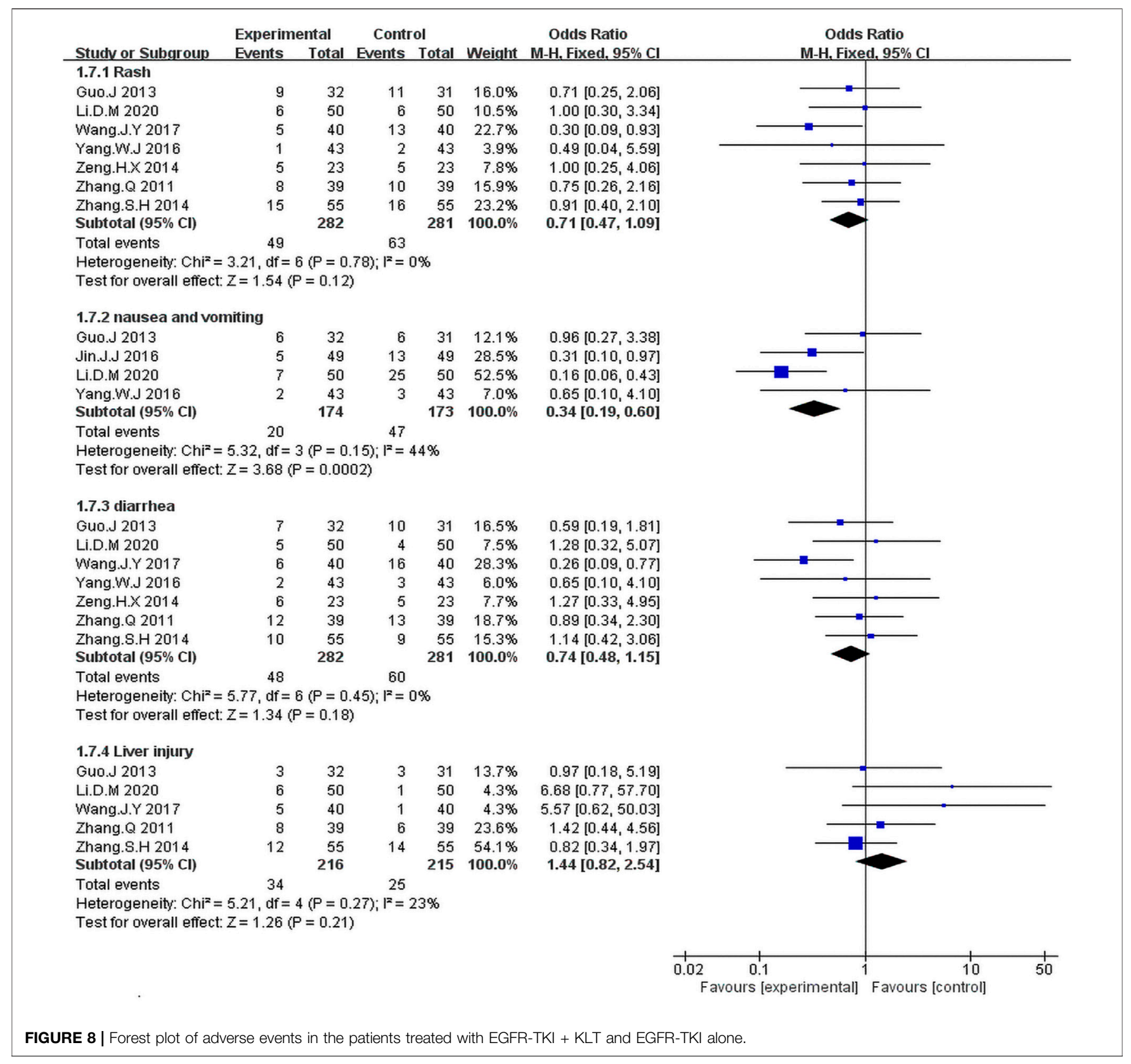

TABLE 3 | Main Anticancer Mechanism of Coix seed in treatment of lung cancer.

\section{Pharmacological effects}

Promotes tumor cell apoptosis Improves the immunity

Inhibits migration and invasion

Inhibits proliferation
Types

NSCL A549 cells Lewis lung carcinoma Lewis lung carcinoma NSCL A549 cells NSCL A549 cells Serum sample
Main anticancer mechanism

Fraction

Mechanism of the intrinsic mitochondrial pathway (Lu et al., 2013) Regulates the expression of NF-кB//kB, increases IL-2 (Pan et al., 2012) Decreases the TAM levels and improves hypoxia status (Duan, 2018) Inhibits JAK2/STAT3 signaling pathway (Wang and Wang, 2019)

Downregulates the S100A4 (Luo et al., 2017)

Reduces the expressions of miRNA-21 (Wu et al., 2018)
Polysaccharide Coix seed extract Coix seed extract Coix seed extract Polysaccharide Coix seed extract of increased percentage of CD4+cells (WMD $=5.36 ; 95 \% \mathrm{CI}$ : $\left.3.60-7.13 ; p<0.00001 ; \mathrm{I}^{2}=91 \%\right)$ and the $\mathrm{CD} 4+/ \mathrm{CD} 8+$ ratio $\left(\mathrm{WMD}=0.18 ; 95 \%\right.$ CI: $\left.0.08-0.27 ; p=0.004 ; \mathrm{I}^{2}=65 \%\right)($ Figure 7$)$.
Four trials (Zeng et al., 2014; Ning et al., 2015; Yang, 2016; Yang et al., 2016) including 382 patients reported percentage of NK cells. The results showed that the KLT plus EGFR-TKI group 


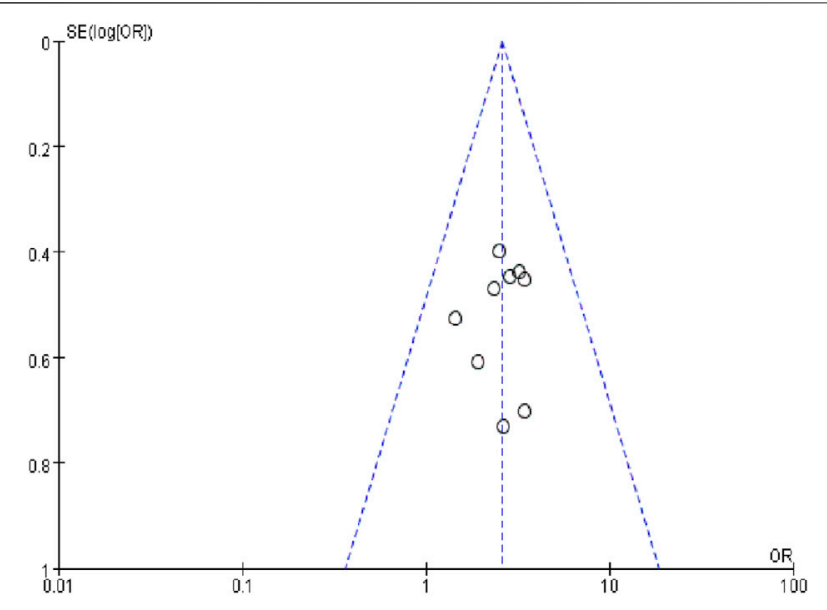

FIGURE 9 | Funnel plot of publication bias.

had an advantage of increased NK cells (WMD $=4.84 ; 95 \% \mathrm{CI}$ : $3.66-6.02 ; p<0.00001 ; \mathrm{I}^{2}=42 \%$ ) (Figure 7).

\section{Adverse Event}

The fixed-effects meta-analysis revealed a significantly lower occurrence rate of nausea and vomiting in the KLT plus EGFR-TKI group than in the control group ( $\mathrm{OR}=0.37 ; 95 \%$ CI:0.16-0.86; $p=0.02 ; \mathrm{I}^{2}=44 \%$ ) (Figure 8).

We identified seven studies (Zhang and Yuan, 2011; Guo and Wang, 2013; Zeng et al., 2014; Zhang and Zhou, 2014; Yang, 2016; Yang et al., 2016; Wang et al., 2017) including 563 patients with rash and diarrhea. The Fixed-effects model revealed that the KLT plus EGFR-TKI group showed no significant differences in the occurrence rates of rash $(\mathrm{OR}=0.71 ; 95 \%$ CI:0.47-1.09; $\left.p=0.12 ; \mathrm{I}^{2}=0 \%\right)$ and diarrhea $(\mathrm{OR}=0.74 ; 95 \%$ CI:0.48-1.15; $\left.p=0.18 ; \mathrm{I}^{2}=0 \%\right)$ compared with the control group (Figure 8).

As can be seen in Figure 8, five studies (Zhang and Yuan, 2011; Guo and Wang, 2013; Zhang and Zhou, 2014; Wang et al., 2017; Li, 2020) including 431 patients reported the incidence of liver injury. The meta-analysis revealed that the combination treatment with KLT plus EGFR-TKI did not significantly reduce the risk of liver injury risk when compared with the EGFR-TKI alone $\left(\mathrm{OR}=1.44 ; 95 \% \mathrm{CI}: 0.82-2.54 ; p=0.21 ; \mathrm{I}^{2}=23 \%\right)$.

\section{Sensitivity Analysis}

We performed a subgroup analysis to examine the source of the heterogeneities in ORR and DCR with regard to sample size, form of KLT, duration of therapy. As shown in Table 2, there were no significant differences between sample size, form of KLT, and therapy duration. Moreover, in terms of DCR index, KLT injection plus EGFR-TKI was found to be more effective than KLT capsule plus EGFR-TKI.

\section{Publication Bias}

A funnel plot of the nine studies that reported ORR data was used to assess publication bias (Figure 9). The funnel plot was asymmetrical, indicating the existence of publication bias.

\section{DISCUSSION}

Clinically, KLT has synergistic effects with radiotherapy and chemotherapy, it has been used in the adjuvant treatment of various tumors such as lung cancer (Hailang et al., 2020a; Li et al., 2020), breast Cancer (Liu S. et al., 2021), hepatocellular carcinoma (Liu et al., 2019; Dou et al., 2020)and colorectal cancer (Mao et al., 2020). The main effects targeted the improvements of clinical efficacy, performance status, KPS score, immune function and the reduction of the side effects of radiotherapy and chemotherapy. In advanced NSCLC, KLT injection combined with platinum-based chemotherapy showed significantly higher efficacy (Wen et al., 2020). EGFR-TKIs have proved effective as first-or second-line therapy for advanced NSCLC. Although the achieved efficacy is significant, most patients acquire resistance to the EGFR-TKIs, which limits the benefits of this treatment. Chinese herbal extracts play a role in overcoming EGFR-TKI resistance in NSCLC (Lee et al., 2021). TCM combined with EGFR-TKI treatment prolonged progressionfree survival (PFS) in patients with NSCLC who were harboring EGFR mutations and caused no adverse effects (Jiao et al., 2019; Tang et al., 2019). TCMs have better effects on ORR than EGFRTKI alone in the treatment of NSCLC (Sui et al., 2020). A systematic review revealed that combination of KLT injection with gefitinib may enhance the therapeutic effectiveness for the patients with NSCLC (Hailang et al., 2020b). However, this study only included seven RCTs up to 2016. The meta-analysis did not include other EGFR-TKIs and different forms of KLT. In recent years, the number of published clinical studies on KLT combined with EGFR-TKI has been increasing. Based on this, we comprehensively searched the literature (up to July 10, 2021) for studies that included icotinib, erlotinib and the capsule form of KLT to systematically evaluate the efficacy and safety of the treatment for stage III/IV non-small cell lung cancer. DCR is regarded as one of the best indicators for predicting OS and PFS (Claret et al., 2014). Our study included DCR as the main outcome indicator. Compared with previous studies, we followed the PRISMA guidelines and additionally performed a subgroup analysis, risk-of-bias assessment, and publication bias analysis for a more comprehensive research.

The meta-analysis was performed with 12 RCTs to evaluate the clinical efficacy of the addition of KLT to EGFR-TKI. The study results showed that compared with EGFR-TKI alone, the combination of KLT and EGFR-TKI significantly improved DCR, ORR, KPS and immune function. The percentages of $\mathrm{CD}^{+}$cells, $\mathrm{NK}$ cells and the $\mathrm{CD} 4+/ \mathrm{CD} 8+$ ratio were significantly increased when KLT was administered, indicating that the immune function of NSCLC patients was improved. Previous studies have shown that KLT has pronounced immunostimulatory activities in C57BL/6 mice (Pan et al., 2012). KLT in combination with chemotherapy influences the peripheral blood $\mathrm{T}$ lymphocyte subsets and blood immunoglobulins in patients with advanced NSCLC (Wen et al., 2020). In the subgroup analysis, no significant differences in ORR and DCR were found between sample size, form of KLT, and therapy duration. This suggests that the dosage form of KLT should be chosen depending on the patient's condition. Moreover, in terms 
of DCR index, KLT injection plus EGFR-TKI was found to be more effective than KLT capsule plus EGFR-TKI. The subgroup analysis revealed that even with the increased treatment duration (from 3 to 9 weeks), DCR was not improved, indicating that 3 weeks of KLT therapy might be an optimal choice. All these results indicate that the addition of KLT might enhance the curative effects of treatment for stage III/IV non-small cell lung cancer.

Although the combination of KLT and gefitinib has shown better clinical efficacy, the synergistic mechanisms are currently unclear. Previous basic experiments showed that the clinical mechanisms of action of KLT for lung cancer are related to the promotion of cancer cell apoptosis (Lu et al., 2013), improvement of the immunity (Pan et al., 2012; Duan, 2018), inhibition of migration and invasion (Luo et al., 2017; Wang and Wang, 2019) and inhibition of proliferation (Wu et al., 2018). (Table 3). KLT combined with Gefitinib can reduce the number of angiogenesis in Lewis lung cancer tissues. The vascular endothelial growth factorkinase domain receptor (VEGF-KDR) pathway may be one of the mechanisms to inhibit tumor angiogenesis. This may be one of the reasons for the synergistic effect in the treatment of lung cancer (Shen et al., 2013). The combination of KLT and gefitinib can induce cell apoptosis significantly. KLT may increase the sensitivity of the human lung adenocarcinoma cell line A549 to gefitinib, (Zhao et al., 2015). At present, the synergistic mechanism of KLT and EGFR-TKI is still unclear, and more experimental data are needed to clarify this.

There were some limitations in this study. First, all the trials were published in China, which is a source of selection bias. Only one study on KLT treatment for lung cancer in the United States is registered at ClinicalTrials.gov (NCT01640730). Second, the studies included in this meta-analysis used an "A + B vs. B"design, without a rigorous control for placebo effect. Third, none of the trials reported the OS and PFS rates. Whether KLT plus EGFRTKI improve the OS and PFS for NSCLC remains unclear. Fourth, only first-generation EGFR-TKIs were used in the studies included in this meta-analysis. No relevant clinical study has used KLT combined with afatinib or osimertinib. Furthermore, our current study has not been registered, and bias may have been present. Future trials are needed to ensure that the reporting follows the consolidated standards for reporting trials guidelines. Additional double-blind, well-

\section{REFERENCES}

Claret, L., Gupta, M., Han, K., Joshi, A., Sarapa, N., He, J., et al. (2014). Prediction of Overall Survival or Progression Free Survival by Disease Control Rate at Week 8 Is Independent of Ethnicity: Western versus Chinese Patients with First-Line Non-small Cell Lung Cancer Treated with Chemotherapy with or without Bevacizumab. J. Clin. Pharmacol. 54(3), 253-257. doi:10.1002/ jcph.191

Dou, D., Zhang, Z. Y., Wu, Z. Y., Qiu, X. D., and Zhong, X. G. (2020). Aidi Injection, Compound Kushen Injection, or Kanglaite Injection: Which Is the Best Partner with Systemic Chemotherapy for Patients with HCC? A Network Meta-Analysis. Evid. Based Complement. Alternat Med. 2020, 5497041. doi:10.1155/2020/5497041

Duan, G. C. (2018). The Effects of Combination of Coix Seed Extract and Cisplatin on TAM and Expression of HIF-1a In Vivo in Lewis Lung Carcinoma. Iran. J. Public Health. 47(6), 838-843. designed and multicenter RCTs are required to confirm the efficacy and safety of this combination treatment.

\section{CONCLUSION}

The results of this study indicate that treatment with KLT combined with EGFR-TKI is more effective than EGFR-TKI alone in the treatment for stage III/IV non-small cell lung cancer patients. KLT can be used as a complementary therapy for NSCLC. However, the low quality of some of the included publications increased the risk of bias, which, to some extent, affected the reliability of the research. The clinical efficacy of KLTmediated adjuvant therapy for stage III/IV non-small cell lung cancer requires verification in methodologically rigorous trials.

\section{DATA AVAILABILITY STATEMENT}

The original contributions presented in the study are included in the article/supplementary material, further inquiries can be directed to the corresponding author.

\section{AUTHOR CONTRIBUTIONS}

FK and XL initiated this study. FK and CW performed study selection, data extraction, and data analysis. The article was drafted by YJ and revised by XL. All authors contributed to the article and approved the submitted version.

\section{FUNDING}

This study was funded by the National Natural Science Foundation of China (No. 81403220 and No. 81904151), Tianjin Health and family planning-high level talent selection and training project, Tianjin Science and Technology Plan Projects (No. 17ZXMFSY00190), and Tianjin Traditional Chinese Medicine Research Project, Tianjin health and family planning commission (No. 2017003).

Guo, J., and Wang, N. (2013). Observation of Kanglaite Injection Combined with Erlotinib in the Treatment of Advanced Non-small Cell Lung Cancer. Hebei Med. J. 35(05), 685-686. doi: doi:10.3969/j.issn.1002-7386.2013.05.021

Hailang, H., Jiping, Z., Ailing, C., and Xianmei, Z. (2020b). The Effect of Kanglaite Injection in Combination with Gefitinib versus Gefitinib Alone in Patients with Nonsmall Cell Lung Cancer: A Meta-Analysis. J. Cancer Res. Ther. 16(4), 745, 751. doi:10.4103/jcrt.JCRT_1213_16

Higgins, J. P., Altman, D. G., Gøtzsche, P. C., Jüni, P., Moher, D., Oxman, A. D., Savovic, J., Schulz, K. F., Weeks, L., and Sterne, J. A. (2011). The Cochrane Collaboration's Tool for Assessing Risk of Bias in Randomised Trials. BMJ. 343(oct18 2), d5928. doi:10.1136/bmj.d5928

Hosomi, Y., Morita, S., Sugawara, S., Kato, T., Fukuhara, T., Gemma, A., et al. (2020). Gefitinib Alone versus Gefitinib Plus Chemotherapy for Non-small-cell Lung Cancer with Mutated Epidermal Growth Factor Receptor: NEJ009 Study. J. Clin. Oncol. 38(2), 115-123. doi:10.1200/JCO.19.01488

Huang, X., Wang, J., Lin, W., Zhang, N., Du, J., Long, Z., et al. (2020a). Kanglaite Injection Plus Platinum-Based Chemotherapy for Stage III/IV Non-small Cell 
Lung Cancer: A Meta-Analysis of 27 RCTs. Phytomedicine. 67, 153154. doi:10.1016/j.phymed.2019.153154

Jiao, L., Xu, J., Sun, J., Chen, Z., Gong, Y., Bi, L., et al. (2019). Chinese Herbal Medicine Combined with EGFR-TKI in EGFR Mutation-Positive Advanced Pulmonary Adenocarcinoma (CATLA): A Multicenter, Randomized, Double-Blind, Placebo-Controlled Trial. Front. Pharmacol. 10, 732. doi:10.3389/fphar.2019.00732

Jin, J. J., Hu, Q. L., and Tao, Q (2016). Effects of Kanglaite Injection Combined with Gefitinib on Inflammatory Factors and Immune Function with Advanced Lung Cancer. Chin. J. Biochem. Pharmaceuticals. 36(12), 147-150.

Lee, H. Y. J., Meng, M., Liu, Y., Su, T., and Kwan, H. Y. (2021). Medicinal Herbs and Bioactive Compounds Overcome the Drug Resistance to Epidermal Growth Factor Receptor Inhibitors in Non-small Cell Lung Cancer. Oncol. Lett. 22(3), 646. doi: doi:10.3892/ol.2021.12907

Li, D. M. (2020). Evaluation of the Clinical Effect of Gefitinib Tablets Combined with Kanglaite Capsules in the Treatment of Stage IIIB/IV Non-small Cell Lung Cancer. Chin. J. Mod. Drug Appl. 14(05), 132-134. doi:10.14164/j.cnki.cn115581/r.2020.05.060

Li, J., Li, H. Z., Zhu, G. H., Gao, R. K., Zhang, Y., Hou, W., et al. (2020). Efficacy and Safety of Kanglaite Injection Combined with First-Line Platinum-Based Chemotherapy in Patients with Advanced NSCLC: a Systematic Review and Meta-Analysis of 32 RCTs. Ann. Palliat. Med. 9(4), 1518-1535. doi:10.21037/ apm-20-616

Liu, J., Liu, X., Ma, J., Li, K., and Xu, C. (2019). The Clinical Efficacy and Safety of Kanglaite Adjuvant Therapy in the Treatment of Advanced Hepatocellular Carcinoma: A PRISMA-Compliant Meta-Analysis. Biosci. Rep. 39(11). doi:10.1042/BSR20193319

Liu, S.-H., Chen, P.-S., Huang, C.-C., Hung, Y.-T., Lee, M.-Y., Lin, W.-H., Lin, Y.C., and Lee, A. Y.-L. (2021a). Unlocking the Mystery of the Therapeutic Effects of Chinese Medicine on Cancer. Front. Pharmacol. 11. doi:10.3389/ fphar.2020.601785

Liu, S., Wang, H., Wang, M., Hu, X., Yang, W., Jin, R., et al. (2021b). Comparative Efficacy and Safety of Chinese Herbal Injections Combined with Cyclophosphamide and 5-Fluorouracil Chemotherapies in Treatment of Breast Cancer: A Bayesian Network Meta-Analysis. Front. Pharmacol. 11. doi:10.3389/fphar.2020.572396

Lu, X., Liu, W., Wu, J., Li, M., Wang, J., Wu, J., et al. (2013). A Polysaccharide Fraction of Adlay Seed (Coixlachryma-Jobi L.) Induces Apoptosis in Human Non-small Cell Lung Cancer A549 Cells. Biochem. Biophys. Res. Commun. 430(2), 846-851. doi:10.1016/j.bbrc.2012.11.058

Luo, C., Wang, X., An, C., Hwang, C. F., Miao, W., Yang, L., et al. (2017). Molecular Inhibition Mechanisms of Cell Migration and Invasion by Coix Polysaccharides in A549 NSCLC Cells via Targeting S100A4. Mol. Med. Rep. 15(1), 309-316. doi: doi:10.3892/mmr.2016.5985

Mao, W., Fan, Y., Cheng, C., Yuan, X., Lan, T., Mao, K., et al. (2020). Efficacy and Safety of Kanglaite Injection Combined with Chemotherapy for Colorectal Cancer: A Protocol for Systematic Review and Meta-Analysis. Medicine (Baltimore). 99(39), e22357. doi:10.1097/MD.0000000000022357

Ni, M., Wang, H., Wang, M., Zhou, W., Zhang, J., Wu, J., et al. (2020). Investigation on the Efficiency of Chinese Herbal Injections for Treating Non-small Cell Lung Cancer with Vinorelbine and Cisplatin Based on Multidimensional Bayesian Network Meta-Analysis. Front. Pharmacol. 11, 631170. doi: doi:10.3389/fphar.2020.631170

Ning, J. L., Zhu, F. Q., and Gao, Y. C. (2015). Effect of Gefitinib Combined with KLT Nepalese on the Function and Quality of Life of Non-small Cell Lung Cancer Patients. MODERN ONCOLOGY. 23(14), 1976-1979. doi:10.3969/ j.issn.1672-4992.2015.14.11

Pan, P., Wu, Y., Guo, Z. Y., Wang, R., Wang, Y. J., and Yuan, Y. F. (2012). Antitumor Activity and Immunomodulatory Effects of the Intraperitoneal Administration of Kanglaite In Vivo in Lewis Lung Carcinoma. J. Ethnopharmacol. 143(2), 680-685. doi: doi:10.1016/j.jep.2012.07.025

Richards, T. B., Henley, S. J., Puckett, M. C., Weir, H. K., Huang, B., Tucker, T. C., et al. (2017). Lung Cancer Survival in the United States by Race and Stage (2001-2009): Findings from the CONCORD-2 Study. Cancer 123, 5079-5099. doi: doi: $10.1002 /$ encr.31029

Rosell, R., Carcereny, E., Gervais, R., Vergnenegre, A., Massuti, B., Felip, E., et al. (2012). Erlotinib versus Standard Chemotherapy as First-Line Treatment for European Patients with Advanced EGFR Mutation-Positive Non-small-cell
Lung Cancer (EURTAC): a Multicentre, Open-Label, Randomised Phase 3 Trial. Lancet Oncol. 13(3), 239-246. doi:10.1016/S1470-2045(11)70393-X

Shah, R. R., and Shah, D. R. (2019). Safety and Tolerability of Epidermal Growth Factor Receptor (EGFR) Tyrosine Kinase Inhibitors in Oncology. Drug Saf. 42(2), 181-198. doi:10.1007/s40264-018-0772-x

Shen, F. Q., Wei, S. J., Hong, L., Wang, J. Y., Zhao, N., and Zhang, F. (2013). The Effect of Kanglaite Injection in Combination with Gefitinib on Angiogenesis in Mice with Lewis Lung Cancer. TUMOR. 33(12), 1047-1053. doi:10.3781/ j.issn.1000-7431.2013.12.003

Shi, Q. H., and Chen, G. F. (2013). Clinical Observation of Kanglaite InJection Combined with Gefitinib in the Treatment of Non-small Cell Lung Cancer with EGFR Positive in Medium or Late Stage. Chin. Manipulation Rehabil. Med. 4(4), 76-78.

Sui, X., Zhang, M., Han, X., Zhang, R., Chen, L., Liu, Y., et al. (2020). Combination of Traditional Chinese Medicine and Epidermal Growth Factor Receptor Tyrosine Kinase Inhibitors in the Treatment of Non-small Cell Lung Cancer: A Systematic Review and Meta-Analysis. Medicine (Baltimore). 99(32), e20683. doi:10.1097/MD.0000000000020683

Sung, H., Ferlay, J., Siegel, R. L., Laversanne, M., Soerjomataram, I., Jemal, A., et al. (2021). Global Cancer Statistics 2020: GLOBOCAN Estimates of Incidence and Mortality Worldwide for 36 Cancers in 185 Countries. CA CANCER J. CLIN. 71(3), 209-249. doi:10.3322/caac.21660

Tang, M., Wang, S., Zhao, B., Wang, W., Zhu, Y., Hu, L., et al. (2019). Traditional Chinese Medicine Prolongs Progression-free Survival and Enhances Therapeutic Effects in Epidermal Growth Factor Receptor Tyrosine Kinase Inhibitor (EGFRTKI)Treated Non-small-cell Lung Cancer (NSCLC) Patients Harboring EGFR Mutations. Med. Sci. Monit. 25, 8430-8437. doi:10.12659/MSM.917251

Wang, G., and Wang, F. (2019). Effects of Kanglaite and Erlotinib Combination on Proliferation and Invasion and JAK2/STAT3 Signaling Pathway of Lung Cancer A549 Cells. J. Zhengzhou Univ. 54(03), 418-422. doi:10.13705/j.issn.16716825.2018.05.042

Wang, J., Wang, B., Chu, H., and Yao, Y. (2016). Intrinsic Resistance to EGFR Tyrosine Kinase Inhibitors in Advanced Non-small-cell Lung Cancer with Activating EGFR Mutations. Onco Targets Ther., 9., 3711, 26. doi:10.2147/ OTT.S106399

Wang, J. Y., Wei, S. J., Hong, L., Zhang, F., Liu, C. Y., and Kong, Y. (2017). Clinical Trial of Gefitinib Tablets Combined with Kanglaite Capsules in the Treatmentof Stage IIIB/IV Non-small Cell Lung Cancer. Chin. J. Clin. Pharmaco 33(17), 1631-1633.

Wen, J., Yang, T., Wang, J., Ma, X., Tong, Y., and Zhao, Y. (2020). Kanglaite Injection Combined with Chemotherapy versus Chemotherapy Alone for the Improvement of Clinical Efficacy and Immune Function in Patients with Advanced Non-smallcell Lung Cancer: A Systematic Review and Meta-Analysis. Evid. Based Complement. Alternat Med. 2020, 8586596. doi:10.1155/2020/8586596

Wu, Y., Zhang, J., Hong, Y., and Wang, X. (2018). Effects of Kanglaite Injection on Serum miRNA-21 in Patients with Advanced Lung Cancer. Med. Sci. Monit. 24, 2901-2906. doi: doi:10.12659/MSM.909719

Xiang, Y., Guo, Z., Zhu, P., Chen, J., and Huang, Y. (2019). Traditional Chinese Medicine as a Cancer Treatment: Modern Perspectives of Ancient but Advanced Science. Cancer Med. 8(5), 1958-1975. doi: doi:10.1002/cam4.2108

Yang, L., Tian, Y., Yang, H., Zhang, L., and Jin, R. (2016). Influence of Kanglaite Combined with Gefitinib on Immune Function and Quality of Life of Patients with Advanced Non-small Cell Lung Cancer. Prog. Mod. Biomed. 16(19), 3728-3730+3775. doi:10.13241/j.cnki.pmb.2016.19.033

Yang, M., Zhu, S.-j., Shen, C., Zhai, R., Li, D.-d., and Fang, M. (2021). Clinical Application of Chinese Herbal Injection for Cancer Care: Evidence-Mapping of the Systematic Reviews, Meta-Analyses, and Randomized Controlled Trials. Front. Pharmacol. 12. doi:10.3389/fphar.2021.666368

Yang, W. J. (2016). Clinical Study on Kanglaite Injection Combined with Icotinib in Treatment of Non-small Cell Lung Cancer. Drugs \& Clinic. 31(12), 1984-1987. doi: doi:10.7501/j.issn.1674-5515.2016.12.027

Zeng, X. H., Zeng, C. S., Liu, L. B., Huang, Z. C., Guo, S. J., and Xu, Q. Y. (2014). Influence of Kanglaite Plus Gefitinib on Immune Function and Quality of Life in Patients with Advanced Non-small-cell Lung Cancer. J. Chin. Pract. Diagn. Ther. 28(09), 930-931.

Zhang, Q., and Yuan, H. (2011). Clinical Observation on 78 Cases of Kanglaite Injection Combined with Gefitinib in the Treatment of Advanced Nonsmall Cell Lung Cancer. Tumor. 31(01), 89-90. doi:10.3781/j.issn.10007431.2011.01.019 
Zhang, S. H., and Zhou, L. X. (2014). Clinical Efficacy of Gefitinib Combined with LT in Treatment of Advanced Non-small Cell Lung Cancer. MODERN ONCOLOGY. 22(12), 2857-2859. doi:10.3969/j.issn.1672-4992.2014.12.26

Zhao, N., Wei, S. J., Hong, L., Wang, J. Y., Shen, F. Q., and Zhang, F. (2015). Effects of Kanglaite Injection on the Apoptosis Induced by Gefitinib in Lung Adenocarcinoma A549 Cell Line. Chin. Clin. Oncol. 20(01), 1-7.

Zhao, Q. F., Shen, H. R., and Zhang, Y. W. (2019). Effects of Gefitinib Combined with Kanglaite Injection on Advanced Lung Cancer in Older Patients. Int. J. Geriatr. 40(5), 257-260. doi:10.3969/j.issn.1674-7593.2019.05.001

Conflict of Interest: The authors declare that the research was conducted in the absence of any commercial or financial relationships that could be construed as a potential conflict of interest.
Publisher's Note: All claims expressed in this article are solely those of the authors and do not necessarily represent those of their affiliated organizations, or those of the publisher, the editors and the reviewers. Any product that may be evaluated in this article, or claim that may be made by its manufacturer, is not guaranteed or endorsed by the publisher.

Copyright (c) 2021 Kong, Wang, Li and Jia. This is an open-access article distributed under the terms of the Creative Commons Attribution License (CC $B Y$ ). The use, distribution or reproduction in other forums is permitted, provided the original author(s) and the copyright owner(s) are credited and that the original publication in this journal is cited, in accordance with accepted academic practice. No use, distribution or reproduction is permitted which does not comply with these terms. 